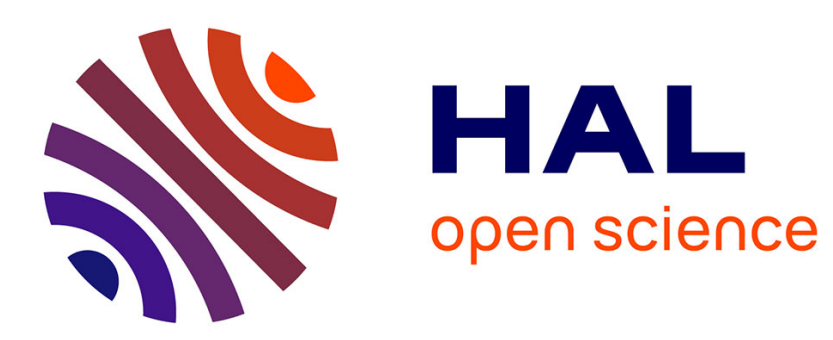

\title{
Multi-level A Priori Hyper-Reduction of mechanical models involving internal variables
}

David Ryckelynck, Djamel Missoum-Benziane

\section{To cite this version:}

David Ryckelynck, Djamel Missoum-Benziane. Multi-level A Priori Hyper-Reduction of mechanical models involving internal variables. Computer Methods in Applied Mechanics and Engineering, 2010, 199, pp.1134-1142. 10.1016/j.cma.2009.12.003 . hal-00461492

HAL Id: hal-00461492

https://hal-mines-paristech.archives-ouvertes.fr/hal-00461492

Submitted on 4 May 2010

HAL is a multi-disciplinary open access archive for the deposit and dissemination of scientific research documents, whether they are published or not. The documents may come from teaching and research institutions in France or abroad, or from public or private research centers.
L'archive ouverte pluridisciplinaire HAL, est destinée au dépôt et à la diffusion de documents scientifiques de niveau recherche, publiés ou non, émanant des établissements d'enseignement et de recherche français ou étrangers, des laboratoires publics ou privés. 


\title{
Multi-level a priori hyper reduction of mechanical models involving internal variables
}

\author{
D. Ryckelynck, D. Missoum Benziane \\ MINES ParisTech, Centre des matériaux, \\ CNRS UMR 7633, BP 8791003 Evry Cedex, France
}

December 1, 2009

\begin{abstract}
This paper concerns the adaptation of reduced-order models during simulations of series of elastoviscoplastic problems. In continuation with previous works, this paper aimed at extending the A Priori Hyper Reduction method (APHR method) for nonlinear thermal problems to nonlinear mechanical problems involving internal variables. This method is an a priori approach because full incremental responses of detailed models are not forecasted in order to build reduced-order models. The recent extension of the Hyper Reduction method to reduction of mechanical models involving internal variables makes possible the reduction of degrees of freedom and the reduction of integration points. A multi-level formulation is introduced to focus on the capability of the method to perform efficient parallel computations to adapt reduced-order models.
\end{abstract}

Keywords: APHR method, POD, parallel computing, FETI, domain decomposition, viscoplasticity.

\section{Introduction}

Elastoviscoplastic models are widely used for fatigue life prediction or crack growth of metallic components $[1,2,3]$. Usually, the constitutive laws are described in the framework of the irreversible thermodynamic processes. The strain history is taken into account using internal variables. These variables are the lump sum of the history of material changes. This approach has its roots in the works by Biot [4] ; Ziegler [5]; Germain [6] or Halpen and Nguyen [7] and has proven its ability to cover a broad spectrum of models in viscoelasticity, viscoplasticity, plasticity and also continuum damage mechanics. Examples of such constitutive laws can be found in [8]. To simplify the presentation of the multi-level APHR method, we consider small-displacement small-strain mechanical problems having unique solutions. According to the framework of the irreversible thermodynamic processes, a constitutive law can be defined by a choice 
of: internal variables $\mathbf{z}$, a free energy $w(\varepsilon, \mathbf{z})$ and a pseudo potential of dissipation $\varphi^{*}$ [9] in case of standard formulations. Some conjugated variables $\mathbf{Z}$ are associated to the internal variables $\mathbf{z}$ using a definition of the dissipation. It turns out that the problems related to such mechanical models are nonlinear and time dependent. The Finite Element (FE) method [33], implicit time integration scheme and the Newton Raphson algorithm are usually used for approximately solving this kind of problem.

The development of large FE models increases the need of low order models created by model reduction methods. The availability of Reduced Order Models (ROMs) can greatly facilitate the solution of series of mechanical problems appearing in optimization problems for instance. The formulation of the reduced-equations differs from the formulation of the detailed equations in the choice of the functional space related to the primal state variables. Most of the time, the state variables are considered as a linear combination of known fields. In case of an a posteriori approach, these fields are obtained by solving detailed preliminary problems. In the framework of modal superposition method, these fields are the eigenvectors related to the free-vibration problem. If the preliminary problems provide linearly independent fields, then the coefficients of the linear combination are the reduced-state variables of the ROM. On the contrary, one has to build a reduced-basis of the subspace spanned by the known fields. The Proper Orthogonal Decomposition (POD) aimed to create such a basis from a set of time dependent fields. The POD model reduction method has been used in a wide range of nonlinear incremental simulations and optimization problems in fluid mechanics [10], in materials science [11, 12], in thermal science [13] and real-time surgery simulation [14]. This method comes from the Karhunen-Loève expansion [15, 16] developed for statistical analyses. A POD basis is an optimal basis of the state subspace spanned by forecasted states possibly related to different simulations of the response of the detailed model. The optimality of the POD has been stated in [17]. The snapshot POD proposed by L. Sirovich [18] had an important contribution to the development of POD basis for the reduction of nonlinear incremental problems. Some details about this method are given in Section 4. During the same period, P. Ladevèze proposed a competitive decomposition method dedicated to elastoplastic models named the radial loading decomposition (RLD) [19]. This decomposition is performed by the LATIN method [19] without solving the full incremental detailed model. The LATIN method aims at building a linear problem to forecast the response of the nonlinear detailed model. This linear problem is defined over the entire time interval. Therefore, a time and space separated representation enables to define the decomposition of the forecasted state evolution. Various LATIN algorithms were proposed to solve optimization problems [20, 21] and multiscale problems [22]. The ***similarities*** between the RLD and the POD have been stated in [23]. 
In practice, during the solution of a series of problems, the POD ROM tends to be less convenient as the current problem differs from the preliminary problems. The main drawback of the classical POD and the snapshot POD methods is the lack of an adaptive procedure in order to modify the ROM basis according to an error indicator. A first adaptive procedure was proposed in [23] using the LATIN method. But the non-incremental scheme used in the LATIN to build linear problems does not facilitate the extension of the method to any mechanical constitutive law. An a posteriori error estimation and a basis adaptation have been proposed in [24] for the reduction of nonaffine-parametrized linear elliptic partial differential equations. This is an interesting approach since a series of nonlinear problems involves a series of parametrized linear problems. But it is no easy task to extend this method to mechanical problems involving internal variables. In most cases, the time is not a simple parameter of the partial differential equations. These difficulties have led us to adopt an incremental approach. Different incremental adaptive strategies were proposed to adapt the reduced-basis approximation: for nonlinear thermal problem [25], for solving some transfer equations [26], for kinetic theory models [27], and for any known state evolution [28]. These papers, excepted the last one, are related to the APHR method using Krylov subspaces in order to extend the subspace spanned by the ROM basis. The recent advance on Hyper Reduction methods proposed in [29] makes possible the extension of the APHR method to the nonlinear mechanical models involving internal variables. But, the expansion using Krylov subspace was not retained. The Krylov approach increases the number of balance residual evaluations. Therefore the computational time devoted to residuals becomes too expensive in case of complex constitutive laws. We propose to extend the ROM basis by using the solution of the FE equations over few time increments. As proposed in [30], a ROM predictor accelerates the Newton-Raphson iterations related to the FE equations.

The multi-level APHR method provides state estimations by summing Finite Element corrections to ROM predictions. The reduced-state variables of the ROM being global and the FE variables being nodal unknowns, the proposed approximation is multi-level. If at the end of a time increment, the ROM prediction is accurate enough, no Finite Element correction is performed. On the contrary, the results of the FE correction make possible to adapt the ROM. When a FE correction has been performed an approximate state evolution is known. Therefore we can apply the adaptive algorithm proposed in [28] dedicated to known state evolutions. The mechanical state is taken into account to expand the subspace spanned by the reduced-basis related to the ROM. To master the growth of the ROM, the adaptive procedure involves a POD of the reduced-state variables. As a result of the Hyper Reduction method [29], a specific spatial integration scheme is introduced when computing the reduced-state variables related to 
the ROM prediction. The constitutive equations are integrated over a Reduced Integration Domain (RID), provided that this equations are local. The quality of the ROM prediction is evaluated thanks to the norm of a truncated residue of the FE balance equations. The computation of the FE correction can be performed using any classical incremental algorithm. It could be a classical Newton-Raphson algorithm or a Newton-Raphson algorithm coupled with a parallel solver such as the FETI method [31] or a mixed domain decomposition method [32].

The paper is organized in the following manner. Section 2 introduces the formulation of the mechanical problem involving internal variables. The formulation of the equations related to the multi-level APHR method is detailed in Section 3. Section 4 reports the numerical results elucidating the usefulness of the proposed method for simplified sensitivity analysis. A modification of a parameter related to the material behaviour and a modification of a geometrical parameter are considered. The results obtained with a sequential solver and those obtained with the FETI parallel solver are discussed in this section. The computational time savings are compared to those obtained with the snapshot POD. Section 5 is the conclusion of this paper. Some indications about the ongoing work are given.

\section{Formulation of the continuous model}

The continuous model of concern is a parametrized mechanical model. We denote $\{p\}$ the column of the model parameters. These parameters can be related to design choices, material coefficients or loading conditions. We consider a series of mechanical problems related to a series of parameter values $\left(\{p\}_{\alpha}\right)_{\alpha=1 \ldots N}{ }^{* * *}$ The various models of the series are supposed to be similar. Therefore, we assume that the potential geometrical modifications can be represented using an unchanged tessellation of the domain with modified positions of vertices. $* * *$ We assume that displacements and strains are small. The stress tensor $\sigma$ is a nonlinear function of the strain history depending on the parameters $\{p\}_{\alpha}$ :

$$
\sigma=\boldsymbol{\Sigma}\left(\varepsilon_{\tau}, \tau \leq t ;\{p\}_{\alpha}\right)
$$

where $\boldsymbol{\Sigma}$ is a formal operator that must be defined by constitutive equations.

The continuous medium is occupying a domain $\Omega\left(\{p\}_{\alpha}\right)$. The nonlinear system is analyzed over a time interval $] 0, T]$. The displacement field at time $\mathrm{t}$ is defined on $\Omega\left(\{p\}_{\alpha}\right)$ and it is denoted by $\mathbf{u}\left(., t,\{p\}_{\alpha}\right)$. The boundary $\partial \Omega\left(\{p\}_{\alpha}\right)$ of $\Omega\left(\{p\}_{\alpha}\right)$ is denoted by $\partial_{U} \Omega\left(\{p\}_{\alpha}\right) \cup \partial_{F} \Omega\left(\{p\}_{\alpha}\right)$. On $\partial_{U} \Omega\left(\{p\}_{\alpha}\right)$, there is the Dirichlet condition $\mathbf{u}\left(., t,\{p\}_{\alpha}\right)=0$ for all $t$. On $\partial_{F} \Omega\left(\{p\}_{\alpha}\right)$, there is a given force field $\mathbf{F}\left(., t,\{p\}_{\alpha}\right)$ 
depending on time $t$ and parameters $\{p\}_{\alpha}$. The displacement field belongs to a function space $\mathcal{V}$ defined by:

$$
\mathcal{V}=\left\{\mathbf{u}\left(., t,\{p\}_{\alpha}\right) \in H^{1}\left(\Omega\left(\{p\}_{\alpha}\right)\right) \mid \mathbf{u}_{\mid} \partial_{U} \Omega\left(\{p\}_{\alpha}\right)=0\right\}
$$

The statement of the mechanical problem is the following. We want to find an estimation of the displacement field $\mathbf{u} \in \mathcal{V}$ defined by the constitutive equations and the principle of virtual work:

$$
\int_{\Omega\left(\{p\}_{\alpha}\right)} \varepsilon\left(\mathbf{u}^{*}\right): \mathbf{\Sigma}\left(\varepsilon(\mathbf{u}), \tau \leq t,\{p\}_{\alpha}\right) d \Omega-\int_{\partial_{F} \Omega\left(\{p\}_{\alpha}\right)} \mathbf{u}^{*} \cdot \mathbf{F}\left(\mathbf{x}, t,\{p\}_{\alpha}\right) d \Gamma=0 \quad \forall \mathbf{u}^{*} \in \mathcal{V}
$$

where $\mathbf{u}^{*}$ is a test function.

In cases of stable materials, the knowledge of the operator $\Sigma$ is sufficient to find a solution of the mechanical balance equations (3). According to the framework of the irreversible thermodynamic processes, a constitutive law can be defined by a choice of: internal variables $\mathbf{z}$, a free energy $w\left(\varepsilon, \mathbf{z},\{p\}_{\alpha}\right)$ and a pseudo potential of dissipation $\varphi^{*}$ [9]. Some conjugated variables $\mathbf{Z}$ are associated to the internal variables $\mathbf{z}$ using the definition of the dissipation. The conjugated variables and the internal variables are connected by the following equation of state:

$$
\mathbf{Z}=-\frac{\partial w}{\partial \mathbf{z}}
$$

Complementary constitutive equations can be proposed without introducing a pseudo potential of dissipation, provided that the Clausius Duhem Inequality is fulfilled ( the rate of entropy production must not be negative):

$$
\dot{\mathbf{z}}=\mathbf{B}\left(\mathbf{Z},\{p\}_{\alpha}\right)
$$

In case of standard formulation of the constitutive equations, the complementary constitutive equations are deduced from the pseudo potential of dissipation $\varphi^{*}\left(\mathbf{Z},\{p\}_{\alpha}\right)$ such that:

$$
\mathbf{B}(\mathbf{Z})=\frac{\partial \varphi^{*}}{\partial \mathbf{Z}}
$$

The initial state of the material is defined by a given initial condition:

$$
\mathbf{z}_{\mid t=0}=\mathbf{z}_{\text {ini }}
$$

The stress $\sigma$ being one of the conjugated variables, the set of equations (4) to (7) defines the operator $\Sigma$. 


\section{The multi-level method}

\subsection{Definition of function spaces}

Three functional subspaces of $\mathcal{V}$ are introduced to facilitate the formulation of the proposed method. The displacement field is forecasted by a ROM prediction and a FE correction. Due to the proposed adaptive procedure the ROM is modified during the incremental treatment of the series of mechanical problems. Therefore we introduce the superscript $(n)$ which denotes the version of the ROM. Using the FE method [33] we obtain the detailed model to be reduced. It involves $m_{h}$ degrees of freedom $\left(q_{j}\left(t,\{p\}_{\alpha}\right)\right)_{j=1 \ldots m_{h}}$. The component $q_{j}$ is a nodal displacement connected to the displacement field by the shape function $\mathbf{N}_{j}$. The FE model is deduced from the continuous model by using a subspace of $\mathcal{V}$ denoted $\mathcal{V}_{h}$ such that :

$$
\begin{array}{r}
\mathcal{V}_{h}=\left\{\mathbf{u} \in \mathcal{V} \mid \exists\{q\} \in \Re^{m_{h}},\right. \\
\left.\mathbf{u}(x)=\sum_{j=1}^{j=m_{h}} \mathbf{N}_{j}\left(x,\{p\}_{\alpha}\right) q_{j} \quad \forall x \in \Omega\left(\{p\}_{\alpha}\right)\right\}
\end{array}
$$

We assume that the topology of the mesh does not depend on the geometrical parameters possibly involved in $\{p\}_{\alpha} . * * *$ To study simple design modifications, only the position of some nodes of the mesh is supposed to change. The list of elements and the list of nodes connected to each element remain unchanged.***

The ROM is defined by a subspace of $\mathcal{V}_{h}$ denoted $\mathcal{V}_{R O M}^{(n)}$ such that:

$$
\begin{array}{r}
\mathcal{V}_{R O M}^{(n)}=\left\{\mathbf{u} \in \mathcal{V}_{h} \mid \exists\{a\}^{(n)} \in \Re^{s},\right. \\
\left.\mathbf{u}(x)=\sum_{k=1}^{k=s} \psi_{k}^{(n)}(x) a_{k}^{(n)} \quad \forall x \in \Omega\left(\{p\}_{\alpha}\right)\right\}
\end{array}
$$

where $\left(\psi_{k}^{(n)}\right)_{k=1 \ldots s}$ are the shape functions of the ROM. They are deduced from the FE shape functions by using a reduction matrix $[A]^{(n)}$ such that :

$$
\psi_{k}^{(n)}=\sum_{j=1}^{j=m_{h}} \mathbf{N}_{j}\left(x,\{p\}_{\alpha}\right) A_{j k}^{(n)} \quad \forall x \in \Omega
$$

As proposed in [29] a RID is introduced to define the balance condition related to the ROM according to the Hyper Reduction method. The basic idea is to select only few nodal balance equations of the FE model in order to state the formulation of the balance condition of the reduced-order model. Therefore only few elements contribute to this formulation. These elements are forming the RID. The most similar idea found in the literature concerns the reduction of chemistry models [34], despite it is not applied on FE models. A mathematical programming based approach provides a selection a few species and chemical reactions in order to reduce the model. 
Let's denote $[\Pi]$ the rectangular matrix selecting the FE balance equations such that $\Pi_{i j}$ is equal to one if the degree of freedom number $j$ is the $i^{\text {th }}$ selected degree of freedom. Else, $\Pi_{i j}$ is equal to zero. A truncated test function $\mathbf{u}_{\Pi}^{*}$ can be associated to any test function $\mathbf{u}^{*}=\mathbf{N} .\{q\}^{*}$ such that

$$
\mathbf{u}_{\Pi}^{*}=\mathbf{N} \cdot[\Pi]^{T} \cdot[\Pi] \cdot\{q\}^{*}
$$

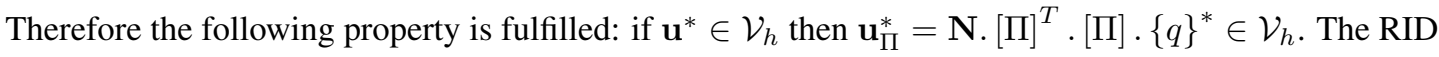
$\Omega_{\Pi}\left(\{p\}_{\alpha}\right)$ is defined as the support of all the truncated test fields:

$$
\begin{aligned}
\Omega\left(\{p\}_{\alpha}\right) & =\bar{\Omega}_{\Pi}\left(\{p\}_{\alpha}\right) \oplus \Omega_{\Pi}\left(\{p\}_{\alpha}\right) \\
\bar{\Omega}_{\Pi}\left(\{p\}_{\alpha}\right) & =\left\{x \in \Omega\left(\{p\}_{\alpha}\right) \mid \forall\{q\}^{*} \in \Re^{n},\left\|\mathbf{u}_{\Pi}^{*}(x)\right\|+\left\|\varepsilon\left(\mathbf{u}_{\Pi}^{*}\right)(x)\right\|=0\right\}
\end{aligned}
$$

where $\left\|\varepsilon\left(\mathbf{u}_{\Pi}^{*}\right)\right\|^{2}=\varepsilon\left(\mathbf{u}_{\Pi}^{*}\right): \varepsilon\left(\mathbf{u}_{\Pi}^{*}\right)$. The hyper reduced-balance condition is obtained using the space function $\mathcal{V}_{\Pi}^{(n)}$ such that:

$$
\begin{array}{r}
\mathcal{V}_{\Pi}^{(n)}=\left\{\mathbf{u} \in \mathcal{V}_{R O M}^{(n)} \mid \exists\{a\}^{(n)} \in \Re\left(\mathbf{u}(x)=\sum_{j=1}^{j=n} \mathbf{N}_{j}(x) q_{j} \quad \forall x \in \Omega,\right. \text { with }\right. \\
\left.\{q\}=[\Pi]^{T} \cdot[\Pi] \cdot[A]^{(n)} \cdot\{a\}^{(n)}\right\}
\end{array}
$$

The hyper reduced-balance condition is the following:

$$
\int_{\Omega_{\Pi}\left(\{p\}_{\alpha}\right)} \varepsilon\left(\mathbf{u}^{*}\right): \mathbf{\Sigma}\left(\varepsilon(\mathbf{u}), \tau \leq t,\{p\}_{\alpha}\right) d \Omega-\int_{\partial_{F} \Omega_{\Pi}\left(\{p\}_{\alpha}\right)} \mathbf{u}^{*} \cdot \mathbf{F}\left(\mathbf{x}, t,\{p\}_{\alpha}\right) d \Gamma=0 \quad \forall \mathbf{u}^{*} \in \mathcal{V}_{\Pi}^{(n)}
$$

where $\partial_{F} \Omega_{\Pi}\left(\{p\}_{\alpha}\right)=\partial_{F} \Omega\left(\{p\}_{\alpha}\right) \cap \Omega_{\Pi}\left(\{p\}_{\alpha}\right)$. This equation involves $s$ scalar equations, where $s$ is the size of the ROM. To obtain a well-posed reduced-problem, the number of selected FE balance equations must be sufficient. It can't be lower than the size of the reduced-basis. A methodology to build this selection of equations is proposed in [29]. Obviously, if all the degrees of freedom are selected to build the RID then the RID covers all the domain $\Omega\left(\{p\}_{\alpha}\right)$. It is clear that the local computations related to such balance condition are restricted to the RID. The equation (15) enables to forecast the reduced-state variables related to the displacement field. The ROM shape functions being global, the displacement field is globally forecasted. But to forecast the entire mechanical state, we must extend the estimated internal variables from the RID to the entire domain. As proposed in [29] the APHR ROM have two bases, one related to the displacements and one related to the internal variables. The second basis is denoted $\left(\Upsilon_{k}^{(n)}\right)_{k=1 \ldots \xi}$. The column of the reduced-internal variables is denoted $\{b\}^{(n)}$. The internal variables are extrapolated from the RID to the full domain by fitting the reduced-internal variables inside the RID. The detailed equations of this extrapolation procedure are given in the next section. 


\subsection{The multi-level incremental algorithm}

The multi-level approximation is the following:

$$
\begin{aligned}
\mathbf{u}\left(x, t,\{p\}_{\alpha}\right)= & \mathbf{u}_{R O M}^{(n)}\left(x, t,\{p\}_{\alpha}\right)+\delta \mathbf{u}_{h}\left(x, t,\{p\}_{\alpha}\right) \quad \mathbf{u}_{R O M}^{(n)} \in \mathcal{V}_{R O M}^{(n)} \quad \delta \mathbf{u}_{h} \in \mathcal{V}_{h} \\
\mathbf{z}\left(x, t,\{p\}_{\alpha}\right)= & \mathbf{z}_{R O M}\left(x, t,\{p\}_{\alpha}\right)+\delta \mathbf{z}\left(x, t,\{p\}_{\alpha}\right) \\
& \text { with } \mathbf{z}_{R O M}\left(x, t,\{p\}_{\alpha}\right)=\sum_{k=1}^{k=\xi} \mathbf{\Upsilon}_{k}^{(n)} b_{k}^{(n)}\left(t,\{p\}_{\alpha}\right)
\end{aligned}
$$

Using a numerical one-step time integration scheme, one can forecast different states of the system at different instants. According to an incremental formulation, the mechanical state is assumed to be known at time $t_{i}$. The unknowns are the state variables at time $t_{i+1}$. Several stages are introduced to forecast the mechanical state over the time increment $\left.] t_{i}, t_{i+1}\right]$ :

- stage one : the reduced-state variables related to the displacement field are forecast with $\delta \mathbf{u}_{h}=0$ and $\delta \mathbf{z}=0$

- stage two : the internal variables are extended using the basis related to these variables

- stage three : an error indicator related to the accuracy of the ROM is evaluated

- stage four : if the ROM prediction is not accurate enough, then the FE correction $\left(\delta \mathbf{u}_{h}, \delta \mathbf{z}\right)$ is computed

- stage five : if the FE correction has been performed, then the reduced-bases are adapted by using the results of the correction stage (stage four).

If the ROM prediction is accurate enough, then no FE computation is performed. In practice, the adaptation of the ROM is achieved only using few FE increments. Moreover, in case of nonlinear equations or parallel computing, the iterative solver takes advantage of the prediction provided by the ROM. The error indicator is the norm of a truncated residue of the FE balance equations. This is the residue of the FE equations selected for the creation of the RID. At the end of stage four, a full state estimation is known. Then the adaptive algorithm [28] devoted to known state evolutions can be applied. The adaptation stage involves an expansion of the subspaces related to the ROM and a selection of the most significant events using a POD of the reduced-state variables. The extension of the bases is provided by orthogonal contributions extracted from the state corrections. During the adaptation of the ROM the reduced-state variables related to the previous computations are updated. A norm of the load magnitude is introduced to define a relative error indicator such that:

$$
\|F\|^{2}=\int_{\partial_{F} \Omega_{\Pi}\left(\{p\}_{\alpha}\right)} \mathbf{F}\left(\mathbf{x}, t,\{p\}_{\alpha}\right) . \mathbf{F}\left(\mathbf{x}, t,\{p\}_{\alpha}\right) d \Gamma
$$


The detailed formulation of the five stages of the algorithm is given below.

Stage one, the unknown are the reduced-state variables $\{a\}^{(n)}\left(t_{i+1},\{p\}_{\alpha}\right)$ and the internal variables $\mathbf{z}_{R O M}\left(x, t_{i+1},\{p\}_{\alpha}\right)$ over $\Omega_{\Pi}\left(\{p\}_{\alpha}\right)$ such that:

$$
\int_{\Omega_{\Pi}\left(\{p\}_{\alpha}\right)} \varepsilon\left(\mathbf{u}^{*}\right): \boldsymbol{\Sigma}\left(\varepsilon\left(\mathbf{u}_{R O M}^{(n)}\right), \tau \leq t,\{p\}_{\alpha}\right) d \Omega-\int_{\partial_{F} \Omega_{\Pi}\left(\{p\}_{\alpha}\right)} \mathbf{u}^{*} \cdot \mathbf{F}\left(\mathbf{x}, t,\{p\}_{\alpha}\right) d \Gamma=0 \quad \forall \mathbf{u}^{*} \in \mathcal{V}_{\Pi}^{(n)}
$$

Stage two, the unknown are the reduced-internal variables $\{b\}^{(n)}\left(t_{i+1},\{p\}_{\alpha}\right)$ and the internal variables $\mathbf{z}_{R O M}\left(x, t_{i+1},\{p\}_{\alpha}\right)$ localized in $\bar{\Omega}_{\Pi}\left(\{p\}_{\alpha}\right)$ such that:

$$
\begin{aligned}
\{b\}^{(n)}\left(t_{i+1},\{p\}_{\alpha}\right) & =\arg _{\min _{\{y\}} H(\{y\})} \\
H(\{y\}) & =\int_{\Omega_{\Pi}\left(\{p\}_{\alpha}\right)}\left\|\mathbf{z}_{R O M}\left(\mathbf{x}, t_{i+1},\{p\}_{\alpha}\right)-\sum_{k=1}^{k=\xi} \mathbf{\Upsilon}_{k}^{(n)}(\mathbf{x}) y_{k}\right\|_{z}^{2} d \Omega \\
\mathbf{z}_{R O M}\left(\mathbf{x}, t_{i+1},\{p\}_{\alpha}\right) & =\sum_{k=1}^{k=\xi} \mathbf{\Upsilon}_{k}^{(n)}(\mathbf{x}) b_{k}^{(n)}\left(t_{i+1},\{p\}_{\alpha}\right) \quad \forall \mathbf{x} \in \bar{\Omega}_{\Pi}\left(\{p\}_{\alpha}\right)
\end{aligned}
$$

Stage three, evaluation of the error indicator $\eta_{R O M}$ related to the truncated residue $\widetilde{\{R\}}$ :

$$
\begin{aligned}
& \widetilde{R}_{j}=\int_{\Omega_{\Pi}\left(\{p\}_{\alpha}\right)} \varepsilon\left(\mathbf{u}^{*}\right): \mathbf{\Sigma}\left(\varepsilon\left(\mathbf{u}_{R O M}^{(n)}\right), \tau \leq t,\{p\}_{\alpha}\right) d \Omega-\int_{\partial_{F} \Omega_{\Pi}\left(\{p\}_{\alpha}\right)} \mathbf{u}^{*} \cdot \mathbf{F}\left(\mathbf{x}, t,\{p\}_{\alpha}\right) d \Gamma(2) \\
& \text { with } \mathbf{u}^{*}=\mathbf{N}_{j}\left(x,\{p\}_{\alpha}\right)\left(\max _{\zeta} \Pi_{\zeta j}\right) \\
& \eta_{R O M}=\|\widetilde{\{R\}}\|
\end{aligned}
$$

Stage four, if $\eta_{R O M}<\epsilon_{R}\|F\|$ then $\delta \mathbf{u}_{h}\left(x, t_{i+1},\{p\}_{\alpha}\right)=0$ and $\delta \mathbf{z}\left(\mathbf{x}, t_{i+1},\{p\}_{\alpha}\right)=0$, else these corrections are such that:

$$
\int_{\Omega\left(\{p\}_{\alpha}\right)} \varepsilon\left(\mathbf{u}^{*}\right): \boldsymbol{\Sigma}\left(\varepsilon\left(\mathbf{u}_{R O M}^{(n)}+\delta \mathbf{u}_{h}\right), \tau \leq t,\{p\}_{\alpha}\right) d \Omega-\int_{\partial_{F} \Omega\left(\{p\}_{\alpha}\right)} \mathbf{u}^{*} \cdot \mathbf{F}\left(\mathbf{x}, t,\{p\}_{\alpha}\right) d \Gamma=0 \quad \forall \mathbf{u}^{*} \in \mathcal{V}_{h}
$$

Stage five, if $\eta_{R O M} \geq \epsilon_{R}$ then the subspace $\mathcal{V}_{R O M}^{(n)}$ can be adapted by using $\delta \mathbf{u}_{h}$. We only consider the residue related to the orthogonal projection of $\delta \mathbf{u}_{h}$ into $\mathcal{V}_{R O M}^{(n)}$. This residue is denoted $\delta_{\perp} \mathbf{u}_{h}$ and it is such that:

$$
\begin{aligned}
& \delta_{\perp} \mathbf{u}_{h}\left(x, t_{i+1},\{p\}_{\alpha}\right)=\delta \mathbf{u}_{h}\left(x, t_{i+1},\{p\}_{\alpha}\right)-\widehat{\delta \mathbf{u}_{h}}\left(x, t_{i+1},\{p\}_{\alpha}\right) \\
& \widehat{\delta \mathbf{u}_{h}}\left(x, t_{i+1},\{p\}_{\alpha}\right)=\sum_{k=1}^{k=s} \psi_{k}^{(n)}(x) \delta a_{k}^{(n)}\left(t_{i+1},\{p\}_{\alpha}\right) \\
& \{\delta a\}^{(n)}\left(t_{i+1},\{p\}_{\alpha}\right)=\operatorname{argmin}_{\{y\}} \int_{\Omega\left(\{p\}_{\alpha}\right)}\left\|\delta \mathbf{u}_{h}\left(x, t_{i+1},\{p\}_{\alpha}\right)-\sum_{k=1}^{k=s} \psi_{k}^{(n)}(x) y_{k}\right\|^{2} d \Omega
\end{aligned}
$$


Then, an extended basis $\left(\psi_{k}^{(n+1 / 2)}\right)_{k=1 \ldots s+1}$ is built preserving the previous predictions such that:

$$
\begin{aligned}
& \psi_{k}^{(n+1 / 2)}=\psi_{k}^{(n)} \quad k \leq s \\
& \psi_{s+1}^{(n+1 / 2)}=\frac{1}{\left\|\delta_{\perp} \mathbf{u}_{h}\right\|} \delta_{\perp} \mathbf{u}_{h} \\
& a_{k}^{(n+1 / 2)}\left(\tau,\{p\}_{\alpha}\right)=a_{k}^{(n)}\left(\tau,\{p\}_{\alpha}\right) \quad \tau \leq t_{i+1} \quad k \leq s \\
& a_{k}^{(n+1 / 2)}\left(\tau,\{p\}_{\beta}\right)=a_{k}^{(n)}\left(\tau,\{p\}_{\beta}\right) \quad \tau \leq T \quad k \leq s \quad \beta<\alpha \\
& a_{s+1}^{(n+1 / 2)}\left(\tau,\{p\}_{\beta}\right)=0 \quad \tau \leq T \quad \beta<\alpha \\
& a_{s+1}^{(n+1 / 2)}\left(\tau,\{p\}_{\alpha}\right)=0 \quad \tau \leq t_{i} \\
& a_{s+1}^{(n+1 / 2)}\left(t_{i+1},\{p\}_{\alpha}\right)=\left\|\delta_{\perp} \mathbf{u}_{h}\right\|
\end{aligned}
$$

A POD decomposition of the reduced-variables is performed to avoid a constant growth of the size of the ROM. This POD consists in finding the vectors $\left(\{V\}_{l}\right)_{l=1 \ldots s+1}$ maximizing the following projection on the forecast reduced-state variables:

$$
\lambda_{l}^{(n+1)}=\frac{\sum_{\beta=1}^{\beta=\alpha-1} \int_{0}^{T}\left(\{a\}^{(n+1 / 2) T}\left(t,\{p\}_{\beta}\right) \cdot\{V\}_{l}\right)^{2} d t+\int_{0}^{t_{j+1}}\left(\{a\}^{(n+1 / 2) T}\left(t,\{p\}_{\alpha}\right) \cdot\{V\}_{l}\right)^{2} d t}{\left\|\{V\}_{l}\right\|^{2}}
$$

within the conditions $\left\|\{V\}_{l}\right\|=1$ and $\lambda_{l} \geq \lambda_{l+1}$. The vectors $\left(\{V\}_{l}\right)_{l=1 \ldots s+1}$ are the eigenvectors of the covariance matrix $[C]^{(n+1 / 2)}$ such that:

$$
\begin{aligned}
{[C]^{(n+1 / 2)}=} & \sum_{\beta=1}^{\beta=\alpha-1} \int_{0}^{T}\{a\}^{(n+1 / 2)}\left(t,\{p\}_{\beta}\right) \cdot\{a\}^{(n+1 / 2) T}\left(t,\{p\}_{\beta}\right) d t \\
& +\int_{0}^{t_{j+1}}\{a\}^{(n+1 / 2)}\left(t,\{p\}_{\alpha}\right) \cdot\{a\}^{(n+1 / 2) T}\left(t,\{p\}_{\alpha}\right) d t
\end{aligned}
$$

The covariance matrix takes into account the previous results to preserve the ability of the ROM to model the related mechanical states. Then, the main events are selected using the following criteria:

$$
[V]=\left[\{V\}_{1}, \ldots,\{V\}_{\widetilde{s}}\right] \quad \text { with } \lambda_{\widetilde{s}}>\epsilon_{P O D} \lambda_{1} \text { and } \lambda_{\widetilde{s}+1} \leq \epsilon_{P O D} \lambda_{1}
$$

$\widetilde{s}$ is the new size of the ROM. At last, the new ROM is a POD reduction of the previous ROM:

$$
\begin{array}{rr}
\psi_{l}^{(n+1)}=\sum_{k=1}^{k=s+1} \psi_{k}^{(n+1 / 2)} V_{k l} & \forall l=1 \ldots \widetilde{s} \\
a_{l}^{(n+1)}\left(t,\{p\}_{\beta}\right)=\sum_{k=1}^{k=s+1} a_{k}^{(n+1 / 2}\left(t,\{p\}_{\beta}\right) V_{k l} & \forall l=1 \ldots \widetilde{s}
\end{array}
$$

A similar adaptive procedure is performed to build $\left(\mathbf{\Upsilon}_{k}^{(n+1)}, b_{k}^{(n+1)}\right)_{k=1 \ldots \xi}$ using $\delta \mathbf{z}_{h}\left(x, t_{i+1},\{p\}_{\alpha}\right)$. This adaptation ends the treatment of the time increment $\left.] t_{i}, t_{i+1}\right]$. 
At the end of each simulation, a last POD of the reduced-state variables is performed. The parameters of the method are the RID and the coefficients $\epsilon_{R}$ and $\epsilon_{P O D}$. The initial ROM

$\left(\left(\psi_{k}^{(0)}, a_{k}^{(0)}\right)_{k=1 \ldots s},\left(\mathbf{\Upsilon}_{k}^{(0)}, b_{k}^{(0)}\right)_{k=1 \ldots \xi}\right)$ can be empty $(s=0$ and $\xi=0)$. In such a case, the first increment of the simulation starts with one full FE increment. The adaptive algorithm proposed in [28] being used, the following property is fullfiled. In case of full integration $\left(\Omega_{\Pi}=\Omega\right)$, the decomposition obtained at the end of the incremental computation is the POD of a state prediction having an accuracy related to $\epsilon_{R}$. The proof of this property can be found in [28].

\subsection{Parallel computing}

The correction stage is a classic FE computation using an initial guess state. Therefore a classical domain decomposition method can be used to solve this problem on a parallel computer. We chose to apply the FETI method [31]. But any mixed method is also convenient [32]. The solution of stage four is provided by a FETI solver coupled to a Newton-Raphson algorithm [36, 35].

A full parallel version of the multi-level APHR method is achieved by using the same domain decomposition for all the stages. Each domain of the decomposition has its own contribution to the RID and its own part of the global shape functions of the ROM. We use the single-program multiple-data approach. Therefore each processor is forecasting the global reduced-state variables. But the computations of contributions to reduced-residuals and to reduced-tangent stiffness matrices are distributed over all the processors. Each processor is summing the contributions of all the other processors. The smaller the ROM size the smaller is the amount of exchange between processors during stage one. Moreover, the POD of the reduced-state variables is duplicated on each processor without any exchange of data between them. ***This method was implemented in the existing Finite Element Analysis software Z-set (www.nwnumerics.com). We modified the linear solver and we extended the data structure of the nonlinear solver ZeBuLon. The new data structure involves the reduction matrix $[A]^{(n)}$ the reduced state variables $\{a\}^{(n)}$ and the index of the ROM version $n$ for the reduced-bases related to displacements and internal variables. The reduction matrix contains nodal values for displacement basis vectors. It contains Gauss point values for internal variable basis vectors. The reduced integration scheme is activated by changing the list of the elements of the mesh.*** 


\section{Numerical results}

\subsection{The elastoviscoplastic constitutive law}

This section aimed to compare the multi-level APHR method, the snapshot POD method, and the FE method. A classic and simple elastoviscoplastic example is considered in order to facilitate the reproduction of the presented results by the reader. The strain tensor is split into the elastic strain tensor and the plastic strain tensor:

$$
\varepsilon=\varepsilon^{p}+\varepsilon^{e}
$$

The accumulated plastic strain $p$ is defined by:

$$
p=\int_{0}^{t} \sqrt{\frac{2}{3} \dot{\varepsilon}_{\tau}^{p}: \dot{\varepsilon}_{\tau}^{p}} d \tau
$$

Two internal variables homogenous in strain are used: the plastic strain tensor $\varepsilon^{p}$ and a tensor $\xi$ related to a kinematic hardening such that:

$$
\mathbf{z}=\left\{\begin{array}{c}
\varepsilon^{p} \\
\xi
\end{array}\right\}, \quad\|\mathbf{z}\|_{z}^{2}=\varepsilon^{p}: \varepsilon^{p}+\xi: \xi
$$

The free energy is assumed to be of the following form (isotropic elasticity):

$$
w\left(\varepsilon, \varepsilon^{p}, \xi\right)=\frac{1}{2}\left(\frac{E}{1+\nu}\left(\varepsilon-\varepsilon^{p}\right):\left(\varepsilon-\varepsilon^{p}\right)+\frac{\nu E}{(1-2 \nu)(1+\nu)}\left(\left(\varepsilon-\varepsilon^{p}\right): \mathbf{I}\right)^{2}\right)+\frac{1}{2} c \xi: \xi
$$

in which $E$ is the Young modulus, $\nu$ is the Poisson ratio and $c$ is a scalar coefficient. $c$ is the first parameter of the mechanical model. The conjugated variables associated to the internal variables are the stress tensor and the kinematic hardening tensor $\Xi$ such that:

$$
\sigma=-\frac{\partial w}{\partial \varepsilon^{p}} \quad, \quad \Xi=-\frac{\partial w}{\partial \xi}
$$

We then obtain the following state laws:

$$
\sigma=\frac{E}{1+\nu} \varepsilon^{e}+\frac{\nu E}{(1-2 \nu)(1+\nu)}\left(\varepsilon^{e}: \mathbf{I}\right) \mathbf{I} \quad, \quad \boldsymbol{\Xi}=-c \xi
$$

The yield surface is defined by the function $f$ :

$$
f(\sigma, \boldsymbol{\Xi})=\left(\left(\sigma^{D}-\boldsymbol{\Xi}\right):\left(\sigma^{D}-\boldsymbol{\Xi}\right)\right)^{1 / 2}-G_{o}
$$

in which $\sigma^{D}$ is the deviatoric part of the stress tensor: $\sigma^{D}=\sigma-\frac{1}{3}\left(\varepsilon^{e}: \mathbf{I}\right) \mathbf{I}$. The complementary constitutive equations are provided by a standard formulation. The pseudo potential of dissipation is such that:

$$
\varphi^{*}(\sigma, \boldsymbol{\Xi})=\frac{K}{\theta+1}\left\langle\frac{f(\sigma, \boldsymbol{\Xi})}{K}\right\rangle_{+}^{\theta+1}
$$


Hence, we obtain the normality rule:

$$
\begin{aligned}
\dot{\varepsilon}^{p} & =\frac{\partial \varphi^{*}}{\partial \sigma}=\dot{p} \frac{\sigma^{D}-\boldsymbol{\Xi}}{\left(\left(\sigma^{D}-\boldsymbol{\Xi}\right):\left(\sigma^{D}-\mathbf{\Xi}\right)\right)^{1 / 2}} \\
\dot{\xi} & =\frac{\partial \varphi^{*}}{\partial \boldsymbol{\Xi}}=-\dot{\varepsilon}^{p} \\
\dot{p} & =\left\langle\frac{f(\sigma, \boldsymbol{\Xi})}{K}\right\rangle_{+}^{\theta}
\end{aligned}
$$

The initial conditions are: $\xi_{\mid t=0}=\varepsilon_{\mid t=0}^{p}=0$.

\subsection{The series of mechanical problems}

The system of interest for sequential computations, is a square plate $(80 \mathrm{~mm} \times 80 \mathrm{~mm} \times 5 \mathrm{~mm}, E=98000$ $\mathrm{MPa}, \nu=0.3, G_{o}=330 \mathrm{MPa}$ ) with a hole in the middle (radius $r$ ). The system of interest for parallel computations, is a square plate $\left(320 \mathrm{~mm} \times 320 \mathrm{~mm} \times 5 \mathrm{~mm}, E=98000 \mathrm{MPa}, \nu=0.3, G_{o}=330 \mathrm{MPa}\right)$ having 16 holes in it (radius $r$ ). In the text below, we refer to the first model as sequential model (SM) and to the second one as the parallel model (PM). Due to the symmetry of both domains and boundary conditions, only one $8^{\text {th }}$ of the plates are modelled. The boundary conditions are shown on Figure 1.

A series of three simulations is considered. The variable parameters of the models are the radius $r$ and the material coefficient $c$ :

$$
\{p\}^{T}=\{r, c\}
$$

The three simulations are related to $\{p\}_{1}^{T}=\left\{r_{1}, c_{1}\right\},\{p\}_{2}^{T}=\left\{r_{1}, c_{2}\right\},\{p\}_{3}^{T}=\left\{r_{2}, c_{1}\right\}$ using $r_{1}=20$ $\mathrm{mm}, r_{2}=22 \mathrm{~mm}, c_{1}=35000 \mathrm{MPa}, c_{2}=28000 \mathrm{MPa}$. $* * *$ To represent the modification of the holes radius by preserving the elements of the mesh, the radial position of the nodes of the mesh is changed such that:***

$$
\begin{aligned}
& \text { if } d_{i}<\widetilde{r} \frac{1}{d_{i}+\Delta d_{i}}=\frac{1}{\widetilde{r}}+\left(\frac{1}{r_{i}}-\frac{1}{\widetilde{r}}\right) \frac{\left(\frac{1}{r_{2}}-\frac{1}{r}\right)}{\left(\frac{1}{r_{1}}-\frac{1}{r}\right)} \\
& \text { if } d_{i} \geq \widetilde{r} \quad \Delta d_{i}=0
\end{aligned}
$$

where $d_{i}$ is the distance between the closest hole center and the node number $i$ and $\widetilde{r}=40 \mathrm{~mm} * * *(\widetilde{r}$ must be greater than the hole radius and smaller than the distance between the hole and the closest external boundary of the domain). Using the mapping defined by equations (54) and (55), the smaller the radius modification the smaller the distances between the new Gauss points and the old Gauss points are. The meshes have always the same number of Gauss points and the same number of nodes. Therefore the transfer of the reduced-bases is straightforward. The reduction matrices related to the reduced-basis remain unchanged. We just take into account node position modifications.*** 
The maximum accumulated plastic strain is reached at point $Q$ shown on Figure 1 for PM. The $\sigma_{22}-\varepsilon_{22}$ curve related to the point Q is shown on Figure 1 for PM. An implicit Euler scheme and a Newton-Raphson algorithm have been chosen for the solution of the nonlinear time dependent equations. The time interval is regularly split into 151 time steps. The SM model involves 3,200 linear hexahedral elements, 332,800 scalar internal variables and 19,926 degrees of freedom related to nodal displacements. The PM model involves 51,200 linear hexahedral elements, 5,324,800 scalar internal variables and 312,942 degrees of freedom related to nodal displacements. Four subdomains have been used to perform the domain decomposition related to the FETI method. These domain decomposition is shown on Figure 1.

The simulations have been performed using the ZeBuLon code [37] and the very efficient DSCPACK linear solver [38] for sequential simulations.

\subsection{Usual strategy using the snapshot POD}

The first FE simulation related to $\{p\}_{1}$ is used has preliminary problem in order to create the snapshots.

These snapshots are the displacements forecast by the FE model at the end of each time step. According to the snapshot POD method the POD basis $\left(\phi_{k}\right)_{k=1 \ldots \gamma}$ is such that:

$$
\begin{aligned}
& \phi_{k}(x)=\sum_{i} \mathbf{u}\left(x, t_{i},\{p\}_{1}\right) d_{i k} \\
& \{d\}_{k} \text { maximize } \mu_{k} \\
& \mu_{k}=\frac{\int_{0}^{T}\left(\int_{\Omega} \mathbf{u}\left(x, t,\{p\}_{1}\right) \cdot \phi_{k} d \Omega\right)^{2} d t}{\int_{\Omega} \phi_{k} \cdot \phi_{k} d \Omega}
\end{aligned}
$$

The key point about the snapshot POD is the reduction of the size of the eigenproblem related to the maximization of $\mu_{k}$ by using a linear combination of the snapshots. The POD basis is used to performed the reduced-simulations related to the parameters $\{p\}_{2}$ and $\{p\}_{3}$. A fixed functional space is therefore introduced to define the POD ROM. This subspace is denoted $\mathcal{V}_{P O D}$. It is such that:

$$
\begin{array}{r}
\mathcal{V}_{P O D}=\left\{\mathbf{u} \in \mathcal{V}_{h} \mid \exists\{a\} \in \Re^{s},\right. \\
\left.\mathbf{u}(x)=\sum_{k=1}^{k=s} \phi_{k}(x) a_{k} \quad \forall x \in \Omega\left(\{p\}_{\alpha}\right)\right\}
\end{array}
$$

The reduced-state variables $\{a\}$ and approximated FE internal variables are defined by the constitutive equations and a classical Galerkin procedure:

$$
\int_{\Omega\left(\{p\}_{\alpha}\right)} \varepsilon\left(\mathbf{u}^{*}\right): \mathbf{\Sigma}\left(\varepsilon(\mathbf{u}), \tau \leq t,\{p\}_{\alpha}\right) d \Omega-\int_{\partial_{F} \Omega\left(\{p\}_{\alpha}\right)} \mathbf{u}^{*} \cdot \mathbf{F}\left(\mathbf{x}, t,\{p\}_{\alpha}\right) d \Gamma=0
$$




$$
\forall \mathbf{u}^{*} \in \mathcal{V}_{P O D} \text { with } \mathbf{u} \in \mathcal{V}_{P O D}
$$

$$
\text { for } \alpha \in\{2,3\}
$$

The constitutive equation being solved over the entire domain, no reduced-basis is required to forecast the internal variables. A prediction of these variables is provided at each integration point of the mesh.

\subsection{Comparison of five strategies}

The purpose of this section is the evaluation of the capability of the ROMs to forecast the modification of the maximum accumulated plastic strain $p_{\max }$ at point $Q$. The reference values are provided by the FE simulations for each $\alpha$ in $\{1,2,3\}$. Therefore the reference strategy consists in three FE simulations. The second strategy is related to the snapshot POD. It involves a preliminary FE simulation and two reduced-simulations using a fixed POD basis as presented in the previous section. The third strategy introduces the adaptive procedure without Hyper Reduction. The three simulations $(\alpha \in\{1,2,3\})$ are performed using the multi-level APHR method with $\Omega_{\Pi}=\Omega, \epsilon_{P O D}=10^{-7}, \epsilon_{R}=0.01$ for the first simulation $(\alpha=1)$ and $\epsilon_{R}=0.1$ for the last simulations $(\alpha \in\{2,3\})$. The fourth strategy introduces the Hyper Reduction method. The third strategy is restarted by changing the reduced-integration domain $\Omega_{\Pi}$. This domain is fixed during the treatment of the three simulations $(\alpha \in\{1,2,3\})$. The RIDs related to the SM and PM models are shown on Figure 1. $\Omega_{\Pi}$ involves 1,083 and 16,640 elements respectively for SM and PM. The fifth strategy is reproducing the fourth using less accurate criteria to adapt the ROM. The parameter $\epsilon_{R}$ have been set equal to 1 during the last simulations ( $\left.\alpha \in\{2,3\}\right)$. These five strategies are denoted respectively FE strategy (FES), POD strategy (PODS), A Priori strategy (APS), accurate APHR strategy (AAPHRS) and fast APHR strategy (FAPHRS).

The size of the ROMs are reported in Table 1. These results are very similar. During the third simulation $(\alpha=3)$, the ROM adaptations produced by APS and AAPHRS increase the size of the ROM. The ROM version correspond to the accumulated amount of FE increments used to extend the APHR ROM. The amount of FE increments related to each series of simulations is shown in Table 2. Each FE increment involves several solution of linear FE problem. The amount of full size linear problems which have been solved is reported in Table 3. Thanks to the initial prevision provided by stage one of the multi-level APHR method the savings related to the amount of global linear problems is bigger than the savings related to the amount of full FE increments. One can observe in Table 2 that only few FE increments were used to adapt the APHR ROMs. 
The five strategies are compared considering various computational times and the accuracy of the maximal accumulated plastic strain. Each series of simulations is performed using the same computer in case of sequential computation or the same set of processors in case of parallel computations. The duration of parallel simulations is given by the processor having the longest computational time. The quality of each simulation is reported in Table 4. The computational time related to each simulation and the total computational time related to each strategy are reported in Table 5. The computational time savings for PM are in good agreement with savings related to the amount of global linear problems. It is not the case for SM because local computations have a greater impact on computational time in case of small size problem. The Hyper Reduction method has a bigger contribution to the computational time savings in such a case.

Let's consider the full series of simulations. Computational time saving must be considered depending on the expected accuracy of the simulations. If the most accurate results are expected then the FE strategy must be used. But APS is also very convenient in such a case and less expansive than FES. Due to the adaptive procedure, APS is the most accurate strategy using a ROM. If the required level of error is $30 \%$, then all the results can be considered. In such a case, the fastest strategy is FAPHRS. But if the required level of error is lower than 10\%, then PODS and FAPHRS are failing to provide convenient results for the third simulation ( $\alpha=3$ ) of the series. In such a case the computational savings provided by PODS and FAPHRS can't be considered. Therefore, the fastest strategy is AAPHRS.

The Hyper Reduction method produces error amplifications. As mentioned in [29], the bigger the RID the smaller are the error amplifications. By increasing the size of the RID the AAPHRS strategy can tends to APS strategy. By decreasing the parameter $\epsilon_{R}$ the FAPHR strategy can tends to APHRS.

Let's consider now each simulation one by one. The maximum computational time saving is $76 \%$ for SM and $97 \%$ for PM. These results are provided by the multi-level APHR methods in case of FAPHRS.

It turns out that the best results concerning the accuracy or concerning the computational time are provided by the multi-level APHR method. APS is less efficient than AAPHRS and FAPHRS. The Hyper reduction technique is very useful to perform fast simulations. The proposed method enables us to choose between accurate simulations or fast simulations. Moreover, one can observe that during the first simulation $(\alpha=1)$ APS, AAPHRS and FAPHRS are more efficient than the FES while the PODS is necessary less 
efficient than FES. This makes the multi-level APHR method very efficient for parallel computing.

\section{Conclusion}

No preliminary finite element solution is needed to build APHR bases. The multi-level APHR method can be considered as a new efficient solver. Even without knowing an initial ROM, the multi-level APHR method is more efficient than FE method. The extension of this solver to parallel or sequential iterative solver is straightforward. This extension has been performed using a parallel FETI solver. Obviously the better initial ROM the faster is the multi-level APHR simulation. Accurate simulations can be performed without using Hyper Reduction. The Hyper Reduction of the equations provides significant computational time savings. Fast simulations can be performed using Hyper Reduction without ROM adaptation. The multi-level APHR method enables to choose gradually between fast or accurate simulations by changing two inputs: the RID and the parameter $\epsilon_{R}$. The best computational saving shown in this paper is $97 \%$. This method is very efficient in case of series of simulations related to modifications of the mechanical model. ***We addressed in this paper simple geometrical modifications by moving some nodes of the mesh. More complex geometrical changes involving remeshing techniques can be certainly taken into account provided that mesh transfer operations are applied to the reduced-basis. The better the reduced-bases are transferred from the previous mesh to the new mesh, the better the quality of the initial ROM will be. Anyway, as mentioned above, if the quality of the transferred ROM (the initial ROM of the new simulation) is too bad, the proposed adaptive algorithm will add the convenient corrections during the new simulation.***

The ongoing works are related to the reduction of models involving damage growth, finite strains and life duration predictions. The case of large number of simulations involved in the series of problems needs deeper research. The extent of the efficiency of the multi-level APHR method remains an open question.

***The APHR method should be very efficient to simulate smooth nonlinear state evolutions. For instance, in case of contact problems (with or without friction), if the contact zones jump from a place to an other at each time increment and if this process is very sensitive to the parameters of the model (stochastic evolution), we can't expect the APHR method to be efficient. But if the contact areas have a smooth evolution in time, the APHR method should be efficient.***

\section{Acknowledgments}

This work is related to the MELOXEL project partially funded by the French National Research Agency (ANR). 


\section{References}

[1] B. Tanguy, J. Besson, R. Piques, A. Pineau, Ductile to brittle transition of an A508 steel characterized by Charpy impact test Part II: modeling of the Charpy transition curve, Engineering Fracture Mechanics, 72 (2005) 413-434.

[2] E. Nicouleau, F. Feyel, S. Quilicy, G. Cailletaud, Structural calculation and lifetime-prediction in thermomechanical fatigue of engine components European Structural Integrity Society, 29 (2002) 331-340.

[3] M. Mazière, J. Besson, S. Forest, B. Tanguy, H. Chalons, F. Vogel, Overspeed burst of elastoviscoplastic rotatig disks - Part I: Analytical and nmerical stability analyses, Eropean Journal of Mechanics A/Solids, 28 (2009) 36-44.

[4] M.A. Biot, Mechanics of Incremental Deformations, Wiley, New York, 1965.

[5] H. Ziegler, Some extremum principles in irreversible thermodynamics with applications to continuum mechanics, In: Sneddon, I.N., Hill, R. (Eds.), Progress in Solid Mechanics, vol. IV. North-Holland, Amsterdam, 1963.

[6] P. Germain, Cours de mecanique des milieux continus, Masson et Cie, Paris, 1973.

[7] B. Halpen, Q.S. Nguyen, Sur les materiaux standards generalises. J. de Mec., 1975; 40:39-63.

[8] J. Lemaitre, J-L. Chaboche, Mecanique des materiaux solides. (1st edn), Dunod: Paris 1985, English version published by Cambridge University Press: cambridge, 1990.

[9] P. Germain, Q. S. Nguyen, P. Suquet, Continuum Thermodynamics, Journal of Applied Mechanics, 50 (1983) 1010-1020.

[10] D. N. Daescu, I. M. Navon, Efficiency of a POD-based reduced second-order adjoint model in 4D-Var data assimilation, International Journal for Numerical Methods in Fluids, Volume 53, Issue 6, (2007) 985-1004.

[11] S. Ganapathysubramanian, N. Zabaras, Design across length scales: a reduced-order model of polycrystal plasticity for the control of microstructure-sensitive material properties, Comput. Methods Appl. Mech. Engrg., 193 (2004)5017-5034.

[12] E. Monteiro, J. Yvonnet, Q. C. He, Computational homogenization for nonlinear conduction in heterogeneous materials using model reduction Computational Materials Science, Volume 42, Issue 4 (2008) 704-712.

[13] O. Balima, Y. Favennec, M. Girault, D. Petit, Comparison between the modal identification method and the POD-Galerkin method for model reduction in nonlinear diffusive systems, International Journal for Numerical Methods in Engineering, 67-7, (2006) 895-915.

[14] S. Niroormandi, I. Alfaro, E. Cueto, F. Chinesta, Real-time deformable models of non-linear tissues by model reduction techniques, Computer Methods and Programs in Biomedicine, 91 (2008) 223-231.

[15] K. Karhunen, Uber lineare methoden in der wahrscheinlichkeitsrechnung, Ann. Acad. Sci. Fennicae, ser. Al. Math. Phys., vol. 37, 1946.

[16] M. M. Loève, Probability theory, The University Series in Higher Mathematics, 3rd Ed, Van Nostrand, Princeton, NJ, 1963.

[17] P. J. Holmes, J. L. Lumley, G. Berkooz, J. Mattingly, R. W. Wittenberg, Low-dimensional models of coherentstructures in turbulence, Physics Reports, 287 (1997) 337-384.

[18] L. Sirovich, Turbulence and the dynamics of coherent structures partI : coherent structures. Quaterly of applied mathematics, XLV, n ${ }^{\circ} 31987$ 561-571. 
[19] P. Ladevèze, Sur une famille d'algorithmes en mècanique des structures, Comptes Rendus Acad. Sci. Paris, Sèrie II, 1985 41-44.

[20] O. Allix, P. Vidal, A new multi-solution approach suitable for structural identification problems, Comput. Methods Appl. Mech. Engrg., 191 (2002) 2727-2758.

[21] P. A. Boucard, S. Buytet, P. A. Guidault, A multiscale strategy for structural optimization, International Journal for Numerical Methods in Engineering, in press.

[22] P. Ladevèze, A. Nouy, On a multiscale computational strategy with time and space homogenization for structural mechanics, Comput. Methods Apll. Mech. Engrg., 192 (2003)3061-3087.

[23] D. Ryckelynck, Réduction a priori de modèles thermomécaniques, C. R. Mécanique, 330 (2002) 499505.

[24] N. C. Nguyen, A posteriori error estimation and basis adaptivity for reduced-basis approximation of nonaffine-parametrized linear elliptic partial differential equations, Journal of Computational Physics, 227 (2007) 983-1006.

[25] D. Ryckelynck, A priori hypereduction method: an adaptive approach, International Journal of Computational Physics, 202 (2005) 346-366.

[26] N. Verdon, C. Allery, D. Ryckelynck, A. Hamdouni, An adaptive ROM approach for solving transfer equations, $15 / 5$ (2006) 589-605.

[27] A. Ammar, D. Ryckelynck, F. Chinesta, R. Keunings, On the reduction of kinetic theory models related to finitely extensible dumbbells Journal of Non-Newtonian Fluid Mechanics, Volume 134, Issues 1-3 (2006) 136-147.

[28] D. Ryckelynck, F. Chinesta, E. Cueto, A. Ammar, On the "a priori" model reduction: Overview and recent developments, Archives of Computational Methods in Engineering, State of the Art Reviews, 13/1 (2006) 91-128.

[29] D. Ryckelynck, Hyper-reduction of mechanical models involving internal variables International Journal for Numerical Methods in Engineering, 77 (2009) 75-89

[30] R. Markovinovic and J. D. Jansen, Accelerating iterative solution methods using reduced-order models as solution predictors, Int. J. Numer. Meth. Engng., 68 (2006) 525-541.

[31] C. Farhat, F.-X. Roux, Implicit pa- rallel processing in structural mechanics. Computational Mechanics Advances, 2 (1994) 1-124.

[32] P. Ladevèze, D. Nèron, P. Gosselet, On mixed and multiscale domain decompositon method, Comput. Methods Apll. Mech. Engrg., 196 (2007) 1526-1540.

[33] O.C. Zienkiewicz, R.L. Taylor. Finite Element Method, vols. 1-3. Butterworth-Heinomann: London, 2000.

[34] I. G. Banerjee, M.G. Lerapetritou, An adaptive reduction scheme to model reactive flow, Combustion and Flame, 144 (2006) 619-633.

[35] F. Feyel, Application du calcul parallèle aux modèles à grand nombre de variables internes, thesis, Ecole Nationale Supérieure des Mines de Paris, 1998.

[36] F. Feyel, S. Calloch, D. Marquis, G. Cailletaud, F.E. Computation of a Triaxial Specimen Using a Polycrystalline Model, CMS, 9 (1997) 141-157.

[37] J. Besson, R. Foerch, Large scale object-oriented finite element code design. Comp. Meth. Appl. Mech. Engrg. 142 (1997) 165-187.

[38] P. Raghavan, Domain-Separator Codes for the parallel solution of sparse linear systems, Tech. Rep. CSE-02-004, Department of Computer Science and Engineering, The Pennsylvania State University, University Park, PA 16802-6106, 2002 

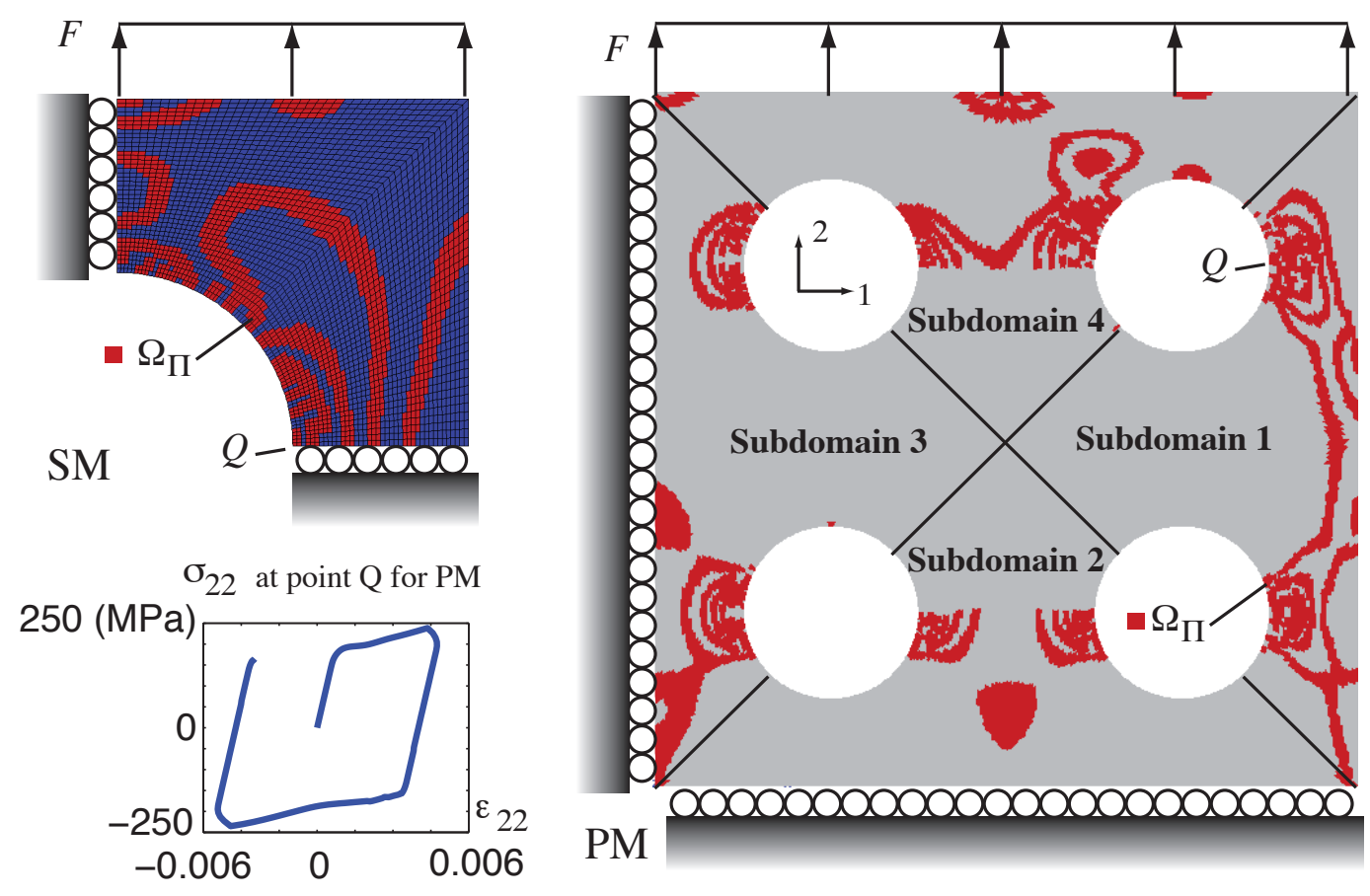

Figure 1: SM and PM models

Table 1: Size of ROMs

$\begin{array}{rccc} & \alpha=1 & \alpha=2 & \alpha=3 \\ \text { for SM } & & & \\ \text { PODS } & & 6 & 6 \\ \text { APS } & 6 & 6 & 7 \\ \text { AAPHRS } & 6 & 6 & 8 \\ \text { FAPHRS } & 6 & 6 & 6 \\ & & & \\ \text { for PM } & & & \\ \text { PODS } & & 7 & 7 \\ \text { APS } & 6 & 6 & 9 \\ \text { AAPHRS } & 6 & 7 & 7 \\ \text { FAPHRS } & 6 & 6 & 6\end{array}$


Table 2: Amount of full FE increments

$\begin{array}{rccccc} & \alpha=1 & \alpha=2 & \alpha=3 & \text { Total } & \text { Saving } \\ \text { for SM } & & & & & \\ \text { FES } & 151 & 151 & 151 & 453 & 0 \% \\ \text { PODS } & 151 & 0 & 0 & 151 & 66 \% \\ \text { APS } & 78 & 7 & 33 & 118 & 74 \% \\ \text { AAPHRS } & 67 & 0 & 24 & 91 & 80 \% \\ \text { FAPHRS } & 67 & 0 & 0 & 67 & 85 \% \\ & & & & & \\ \text { for PM } & & & & & \\ \text { FES } & 151 & 151 & 151 & 453 & 0 \% \\ \text { PODS } & 151 & 0 & 0 & 0 & 66 \% \\ \text { APS } & 78 & 9 & 29 & 116 & 74 \% \\ \text { AAPHRS } & 54 & 0 & 3 & 57 & 87 \% \\ \text { FAPHRS } & 54 & 0 & 0 & 54 & 88 \%\end{array}$

Table 3: Amount of global linear problems

$\begin{array}{rccccc} & \alpha=1 & \alpha=2 & \alpha=3 & \text { Total } & \text { Saving } \\ \text { for SM } & & & & & \\ \text { FES } & 256 & 257 & 292 & 805 & 0 \% \\ \text { PODS } & 256 & 0 & 0 & 256 & 68 \% \\ \text { APS } & 78 & 9 & 58 & 145 & 82 \% \\ \text { AAPHRS } & 71 & 0 & 55 & 126 & 84 \% \\ \text { FAPHRS } & 71 & 0 & 0 & 71 & 91 \% \\ & & & & & \\ \text { for PM } & & & & & \\ \text { FES } & 248 & 247 & 282 & 777 & 0 \% \\ \text { PODS } & 248 & 0 & 0 & 248 & 68 \% \\ \text { APS } & 78 & 11 & 51 & 140 & 82 \% \\ \text { AAPHRS } & 63 & 0 & 4 & 67 & 91 \% \\ \text { FAPHRS } & 63 & 0 & 0 & 63 & 92 \%\end{array}$

Table 4: Relative error between the approximate and the FE maximal accumulated plastic strain

\begin{tabular}{|c|c|c|c|c|}
\hline for SM & $\alpha=1$ & $\alpha=2$ & $\alpha=3$ & maximum \\
\hline PODS & $<0.5 \%$ & $<0.5 \%$ & $26 \%$ & $26 \%$ \\
\hline APS & $<0.5 \%$ & $<0.5 \%$ & $<0.5 \%$ & $<0.5 \%$ \\
\hline AAPHRS & $<0.5 \%$ & $<0.5 \%$ & $9 \%$ & $9 \%$ \\
\hline FAPHRS & $<0.5 \%$ & $<0.5 \%$ & $29 \%$ & $29 \%$ \\
\hline for PM & & & & \\
\hline PODS & $2 \%$ & $<1 \%$ & $17 \%$ & $17 \%$ \\
\hline APS & $2 \%$ & $<1 \%$ & $<1 \%$ & $2 \%$ \\
\hline AAPHRS & $2 \%$ & $3 \%$ & $6 \%$ & $6 \%$ \\
\hline FAPHRS & $2 \%$ & $4 \%$ & $26 \%$ & $26 \%$ \\
\hline
\end{tabular}


Table 5: Computational times

\begin{tabular}{|c|c|c|c|c|c|c|}
\hline for SM & $\alpha=1$ & $\alpha=2$ & $\alpha=3$ & Total & Total CPU Saving & Maximum CPU Saving \\
\hline FES & $601 \mathrm{~s}$ & $603 \mathrm{~s}$ & $719 \mathrm{~s}$ & $1923 \mathrm{~s}$ & $0 \%$ & $0 \%$ \\
\hline PODS & $671 \mathrm{~s}$ & $362 \mathrm{~s}$ & $335 \mathrm{~s}$ & $1368 \mathrm{~s}$ & $29 \%$ & $53 \%$ \\
\hline APS & $583 \mathrm{~s}$ & $389 \mathrm{~s}$ & $589 \mathrm{~s}$ & $1561 \mathrm{~s}$ & $19 \%$ & $35 \%$ \\
\hline AAPHRS & $419 \mathrm{~s}$ & $143 \mathrm{~s}$ & $476 \mathrm{~s}$ & $1038 \mathrm{~s}$ & $46 \%$ & $76 \%$ \\
\hline FAPHRS & $418 \mathrm{~s}$ & $144 \mathrm{~s}$ & $168 \mathrm{~s}$ & $1038 \mathrm{~s}$ & $62 \%$ & $76 \%$ \\
\hline for PM & & & & & & \\
\hline FES & $27067 \mathrm{~s}$ & $25740 \mathrm{~s}$ & $28661 \mathrm{~s}$ & $81468 \mathrm{~s}$ & $0 \%$ & $0 \%$ \\
\hline PODS & $27587 \mathrm{~s}$ & $1524 \mathrm{~s}$ & $1542 \mathrm{~s}$ & $30655 \mathrm{~s}$ & $62 \%$ & $95 \%$ \\
\hline APS & $9914 \mathrm{~s}$ & $2685 \mathrm{~s}$ & $6878 \mathrm{~s}$ & $19477 \mathrm{~s}$ & $76 \%$ & $90 \%$ \\
\hline AAPHRS & $10376 \mathrm{~s}$ & $751 \mathrm{~s}$ & $2995 \mathrm{~s}$ & $14112 \mathrm{~s}$ & $83 \%$ & $97 \%$ \\
\hline FAPHRS & $10376 \mathrm{~s}$ & $724 \mathrm{~s}$ & $732 \mathrm{~s}$ & $11832 \mathrm{~s}$ & $85 \%$ & $97 \%$ \\
\hline
\end{tabular}

